\title{
VARIACIONES ESPACIO-TEMPORALES DE LA FLORA DIATOMOLÓGICA EN Laguna de Los Pozuelos (Jujuy, Argentina)
}

\author{
ANA LUCÍA GONZÁLEZ ACHEM¹', CLAUDIA SEELIGMANN² y MARIELA ALDERETE²
}

\begin{abstract}
Summary: Spatial-temporal variations of diatom flora in Laguna de Los Pozuelos (Jujuy, Argentina). Pozuelos shallow lake is located at the northwest of the province of Jujuy (Argentina) at 3625 masl. It was designated as National Natural Monument and Wetland of International Importance (RAMSAR Convention). The aim of this study was to analyze the spatio-temporal variations of the diatom flora in relation to physicochemical variables and species of Phoenicopteridae. Samples were obtained during 4 field-trips between 2008 and 2010. A total of 116 infrageneric taxa were determined. Eighteen species had a frequency of occurrence greater than $50 \%$, 34 were exclusive of the shallow lake, 32 were exclusive of the Cincel River (tributary of the shallow lake) and 39\% were observed just once. Species richness was more variable in the shallow lake than in the river. The Redundancy Analysis (RDA) based on physico-chemical data and diatom composition allowed discriminating 3 main groups, which were mainly associated with temperature, salinity and $\mathrm{pH}$. There were $31 \mathrm{new}$ records for this aquatic environment and 18 new records for the province of Jujuy.
\end{abstract}

Key words: Bacillariophyceae, Andean wetlands, flemish.

Resumen: La Laguna de Los Pozuelos se ubica al noroeste de la provincia de Jujuy (Argentina) a 3625 msnm. Fue designada Monumento Natural Nacional y Humedal de Importancia Internacional (Convención RAMSAR). Este trabajo tuvo como objetivo analizar las variaciones espacio-temporales de la flora de diatomeas y su relación con variables fisicoquímicos y especies de la familia Phoenicopteridae. Se estudiaron muestras recolectadas en 4 campañas entre 2008-2010. Se determinaron un total de 116 taxones infragenéricos. Dieciocho especies tuvieron una frecuencia de ocurrencia superior al $50 \%, 34$ fueron exclusivas de la laguna, 32 del río Cincel (tributario de la laguna) y un $39 \%$ fue observado una sola vez. La riqueza específica fue más variable en la laguna que en el río. El Análisis de Redundancia (RDA) realizado en base a las variables físico-químicas y composición diatomológica permitió diferenciar 3 grupos principales de especies, que se asociaron principalmente con la temperatura, salinidad y $\mathrm{pH}$. Se registraron por primera vez 31 taxones para la laguna y 18 nuevas citas para la provincia de Jujuy.

Palabras clave: Bacillariophyceae, humedales altoandinos, flamencos.

\section{INTRODUCCIÓN}

Los lagos y lagunas altoandinas son ambientes naturalmente inestables a causa de la marcada estacionalidad climática y las sequías periódicas (Mascitti, 1996). Están sujetos a expansiones y retracciones determinadas por los regímenes de las lluvias, los que producen fluctuaciones notables

${ }^{1}$ Facultad de Cs. Nat. e Inst. M. Lillo UNT, M. Lillo 205, 4000 S. M. de Tucumán, Argentina. CONICET.

2 ILINOA, Facultad de Cs. Nat. e Inst. M. Lillo UNT, M. Lillo 205, 4000 S. M. de Tucumán, Argentina. E-mail: claudiaseeligmann@gmail.com de tamaño y del nivel del agua. Por efecto de ello pueden detectarse grandes variaciones en la concentración salina durante períodos tanto cortos como largos. Como resultados de estos procesos los humedales de altura se caracterizan por su baja predictibilidad, alta fragilidad frente a la acción antrópica y baja resiliencia.

La Laguna de Los Pozuelos fue designada Monumento Natural Nacional en 1980 y a partir de 1990 toda la cuenca que alimenta a la laguna está amparada por la categoría de conservación de Reserva de la Biosfera (RB), en el marco del programa "El Hombre y La Biósfera" (MAB; "Man and the Biosphere") de la UNESCO, cubriendo una superficie de 40.000 ha. En 1992 el Monumento 
Natural Laguna de los Pozuelos fue incluido en la Lista de Humedales de Importancia Internacional y Convención de RAMSAR, declarándose como Sitio Ramsar a las 16.224 ha (bajo la denominación de Laguna de los Pozuelos). A partir de 2005, se la designó sitio de reserva por el Grupo de Conservación de Flamencos Altoandinos (GCFA).

Para Pozuelos se citan 35 especies de aves acuáticas entre las que se encuentran los flamencos Phoenicoparrus andinus ("parina grande"), $P$. jamesi ("parina chica") y Phoenicopterus chilensis ("parina común") que constituyen el grupo dominante (Mascitti \& Castañera, 1991). Además, estas especies de flamencos son comunes a los sistemas hipersalinos de altura de Argentina, Bolivia, Chile y Perú (Diaz \& Maidana, 2005), encontrándose todas ellas con problemas de conservación (Mascitti, 1996). Mascitti (1998) informa que $P$. jamesi habita la laguna en el invierno y primavera junto a $P$. andinus y $P$. chilensis, mientras que durante el verano migra a altitudes más elevadas. La laguna de los Pozuelos es uno de los sitios más importantes para el flamenco andino y el flamenco "puneño o parina chica" durante sus desplazamientos altitudinales, sobre todo en invierno donde la mayoría de las lagunas altoandinas se congelan. Se puede considerar como un sitio importante de concentración y parada de flamencos altoandinos, como hábitat alternativo o complementario respecto a los humedales del norte de Chile y sur de Bolivia, lugares donde se concentran la mayor cantidad de colonias de nidificación de la especie (Rodríguez, 2012).

Hulbert (1982), Hulbert \& Chang (1983) y Mascitti (1998) indican que las diatomeas son el principal componente en la dieta de los flamencos y este último autor señala que existe una preferencia por el tamaño de las mismas independiente de su disponibilidad en la laguna. Caziani \& Derlindati (2000) describieron a las especies $P$. jamesi y $P$. andinus como herbívoras con ingestión especial de diatomeas. El primero filtra presas pequeñas entre 20 a $40 \mu \mathrm{m}$ de largo, mientras que el segundo ingiere especies de hasta $90 \mu \mathrm{m}$ de largo. $P$. chilensis incluye en su dieta organismos más grandes como braquiópodos, anfípodos, copépodos y larvas de insectos.

La Delegación Regional Noroeste de Parques Nacionales para el Sistema de Información de Biodiversidad, manifestó su interés en realizar una planificación de monitoreos de biodiversidad en la laguna, ya que si bien existen algunos antecedentes para este cuerpo de agua (García Fernández \& Tecchi, 1991; Mascitti, 1996; Salusso et al., 1997; Maidana et al., 1998) aún quedan grandes vacíos de información que son necesarios para explicar la diversidad y la abundancia de aves acuáticas en Pozuelos. En función de esto, en 2008 se iniciaron estudios limnológicos en Laguna de los Pozuelos debido a su relevante importancia dentro de los humedales altoandinos.

Esta contribución tuvo como objetivo analizar si las variaciones espaciales (río-laguna) y estacionales (retracción y expansión del espejo de agua) que sufrió la Laguna de los Pozuelos durante los dos años de muestreo tienen incidencia sobre la composición de la flora de diatomeas y si esta influye en la abundancia de las tres especies de flamencos que habitan en este cuerpo de agua.

\section{Área de estudio}

El Bolsón de la Laguna de Los pozuelos (Fig. 1), se sitúa en los departamentos de Yavi, Santa Catalina y Cochinoca, en el norte de la provincia de Jujuy y corresponde a uno de las principales cuencas endorreicas del altiplano argentino.

Laguna de los Pozuelos se encuentra dentro de la región Andina (Morrone, 1999), en una cuenca de drenaje endorreico cuya parte más baja corresponde a este cuerpo de agua $(3625 \mathrm{msnm})$ y la más alta a $4808 \mathrm{msnm}$, al cerro Salli de la Sierra de la Rinconada. El escurrimiento de la cuenca tiene un régimen temporario, que aporta agua superficial solamente en el período estival; se infiltra al llegar a la zona inferior del piedemonte, generando fluctuaciones importantes en el nivel de la laguna durante todo el año como consecuencia de las características climáticas de la zona. La región se caracteriza por su aridez con precipitaciones menores a $350 \mathrm{~mm}$ anuales, por amplitudes térmicas elevadas (más de $18^{\circ} \mathrm{C}$ ) y fuertes vientos. Los ciclos de lluvia-sequía en Pozuelos tienen una extensión temporal de 3 a 4 años, los que producen las expansiones y retracciones estacionales. Rodríguez (2012) señaló que la evolución temporal del tamaño de este cuerpo de agua presenta dos etapas claramente identificables: entre los meses de enero a abril existe un importante aumento de tamaño coincidente con el periodo estival. Posteriormente, entre los meses de mayo a diciembre la tendencia 


\section{A. L. González Achem et al. - Flora diatomológica en Laguna de Los Pozuelos}

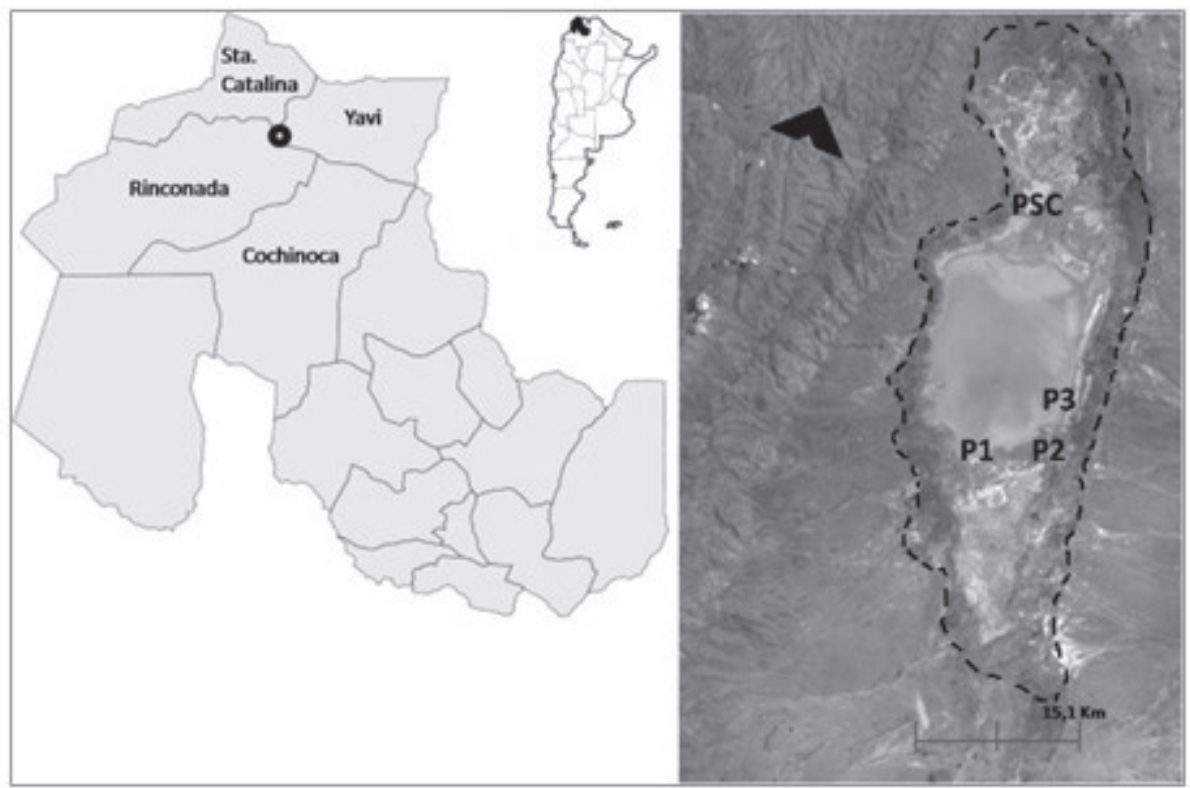

Fig. 1. Mapa de localización de los puntos de muestreo en la Laguna de Los Pozuelos (P1 y P3) y los ríos Cincel (P2) y Santa Catalina (PSC). Se destaca en línea punteada la máxima superficie que alcanzó la laguna.

se invierte para disminuir su área en forma gradual, es decir, que se produce un descenso de más del $30 \%$ de la superficie total general (Fig. 1). También estimó que el periodo del inicio de estiaje comienza durante los meses de invierno y el periodo de recarga, a partir del mes de enero. Frenguelli (1928), a través de un examen climatológico para la Puna de Atacama propuso que los actuales salares han sido cuencas lacustres similares a las que todavía existen en la parte húmeda del Altiplano. Ya desde entonces este autor alertaba sobre una progresiva desertización desde el sudoeste hacia el nordeste como consecuencia de una tendencia a una reducción de las precipitaciones. Posteriormente Cajal (1998) señaló que Laguna de los Pozuelos hasta la década de los años 50, registró ligeras expansiones, fenómeno que no se repitió hasta 1991, ya que desde entonces ocurrieron diversas contracciones, con desecamientos parciales, o casi totales, que repercutieron en la flora y la fauna.

Los principales tributarios de Laguna de Los Pozuelos son el río Cincel por el sur y el río Santa Catalina por el norte. Por este último escurren los caudales de mayor importancia de la cuenca ya que el valor medio registrado para el período 1992-1996 fue de $900 \mathrm{~L} / \mathrm{s}$ para la temporada estival y $300 \mathrm{~L} / \mathrm{s}$ para la época de estiaje. El caudal estimado para el río Cincel fue de $500 \mathrm{~L} / \mathrm{s}$ para el verano y de 100 L/s para la época de estiaje (Rodríguez, 2012). Este tributario atraviesa la mina Pan de Azúcar la que actualmente no está activa y se encuentra a unos 20 $\mathrm{km}$ al sur de la laguna.

Análisis químicos previos a este estudio (Camacho, 2009), permitieron determinar una marcada diferencia entre la salinidad de las aguas de los tributarios con respecto a la de la laguna. De acuerdo a la secuencia de cationes, el sodio fue dominante seguido por el calcio, mientras que el anión más abundante fue el cloro seguido por sulfato.

Fitogeográficamente la cuenca pertenece a la Provincia Puneña (Cabrera \& Willink, 1973). La vegetación está determinada por el clima regional, en la que se diferencia una estepa arbustiva o del tolar con Parastrephia lepidophylla y pastizales graminosos con Festuca orthophylla. La única especie arbórea es la "Queñoa" (Polylepis tomentella). La vegetación perilagunar está compuesta de un césped bajo de gramíneas rizomatosas, Distichilis humilis y cojines Anthobryum triandrum (Mascitti, 1996). 


\section{Materiales y Métodos}

Desde 2008 a 2010 se realizaron cuatro campañas: dos en verano (2009 y 2010) y dos en invierno (2008 y 2009). Se seleccionaron para su estudio 4 sitios: 2 en la laguna (codificados como P1 y P3), un punto para el río Cincel (P2) y uno para el río Santa Catalina (PSC, muestreado sólo en invierno de 2009) (Fig. 1). La falta de agua en invierno o la inaccesibilidad en verano en algunos sitios impidieron realizar el muestreo a lo largo de todo el período y en total se obtuvieron once muestras. Los sitios fueron georeferenciados y se determinó su altitud utilizando un equipo de GPS. Se midieron in situ: temperatura del agua, conductividad eléctrica, oxígeno disuelto, profundidad del cuerpo de agua, sólidos totales y $\mathrm{pH}$. La salinidad fue estimada a partir de los valores de conductividad eléctrica, medidos de acuerdo a Dejoux (1993) (Tabla 1).

Para el análisis diatomológico se extrajeron muestras cualitativas con red de plancton de 20 $\mu \mathrm{m}$ de malla y cuantitativas, colectadas sin filtrar, en envases plásticos de $250 \mathrm{~mL}$. Ambos tipos de muestras fueron tomadas en la zona litoral de la laguna y fijadas con formaldehído al $4 \%$.

Para la eliminación de la materia orgánica se utilizó agua oxigenada (30\%) y calor (Battarbee, 1986). El material así tratado se montó en NAPHRAX ${ }^{\circledR}$ para su observación en microscopio binocular con cámara incorporada para captura de imágenes. La identificación taxonómica se basó, en general, en las monografías de Hustedt (1927, 1930, 1959-1966), Krammer \& Lange-Bertalot (1986, 1988, 1991, 2000, 2004), Lange-Bertalot (1997, 1999, 2001), Patrick \& Reimer (1966, 1975), Round et al. (1990), Rumrich et al. (2000) y obras específicas de autores varios. Para la distribución geográfica en Argentina se consultó a Vouilloud (2003) y trabajos posteriores de la zona (Seeligmann et al., 2008; Maidana et al., 2009, 2011). Los taxones identificados en las muestras analizadas figuran en la Tabla 2.

Las abundancias relativas de diatomeas se calcularon a partir del recuento de un mínimo de 400 valvas por muestra y la diversidad fue estimada a través del Índice de Shannon $(\mathrm{H})$.

La Delegación Regional Noroeste de Parques Nacionales para el Sistema de Información de Biodiversidad proporcionó registros de los censos ornitológicos y cálculos de área de la laguna, realizados en las mismas fechas de muestreo.

\section{Tratamiento estadístico}

Se graficó un índice de dominancia para cada especie de diatomea presente en la laguna (P1) y en el río Cincel (P2). El índice se basó en la abundancia relativa de cada especie y se consideraron aquellas que estuvieron presentes al menos en $5 \%$ de cada muestra. Además, se llevó a cabo el análisis multivariado NMDS (Non metric Multidimensional Scaling) con el fin de ordenar las muestras agrupadas por sitio, temporada y estación del año $(\mathrm{N}=10$, ya que no se incluyó en el análisis estadístico la muestra correspondiente al río Santa Catalina) en función de las variables fisicoquímicas (oxígeno disuelto, $\mathrm{pH}$, temperatura, salinidad, sólidos totales, profundidad) y los valores del índice de diversidad, riqueza de especies y área del cuerpo de agua. La distancia Bray Curtis se seleccionó como medida de similitud y se midió el ajuste de este ordenamiento multivariado mediante el estadístico STRESS. Valores cercanos a cero indicarían que el modelo NMDS seleccionado presenta un "ajuste" aceptable con la matriz de datos observada (James \& Culloch, 1990). Su valoración se midió con el test de aleatorización de Monte Carlo. Se realizó un análisis de correlación de Spearman no paramétrico para medir el grado de asociación lineal entre cada uno de los ejes extraídos del análisis del NMDS y las variables relacionadas a cada una de las muestras.

El análisis de redundancia (RDA) (Legendre \& Legendre, 2000) fue seleccionado para relacionar los datos de abundancia de la matriz de diatomeas a la matriz de variables ambientales. En esta técnica se asume que las especies tienen respuestas lineales a los gradientes ecológicos. Un examen preliminar de los datos mostró que la longitud de los gradientes fue menor que dos, condición recomendada para utilizar el RDA (Ter Braak \& Šmilauer, 1998).

Se seleccionaron para el RDA las especies que estuvieron presentes en al menos un $5 \%$ de las muestras. Las especies infrecuentes de ocurrencia aleatoria pueden producir efectos espurios (Aragón $\&$ Morales, 2003). Para la elección de los ejes de ordenamiento se tuvo en cuenta la proporción de varianza explicada por cada eje, considerándose aquellos con más del $11 \%$ de varianza. Debido a que las abundancias de las especies mostraron asimetría, se procedió a transformación logarítmica (abundancia +1$)$ y las variables ambientales fueron estandarizadas (Ter Braak, 1986). Además 
Tabla 1. Variables fisicoquímicas determinadas para los puntos de muestreo. OD: oxígeno disuelto; CE: conductividad eléctrica; ST: sólidos totales; Prof.: Profundidad; Temp.: Temperatura, pH y Salinidad.

\begin{tabular}{|cccccccc|}
\hline Sitios & OD $(\mathbf{m g} / \mathbf{L})$ & $\mathbf{C . E}(\mathbf{m S} / \mathbf{c m})$ & ST $(\mathbf{m g} / \mathbf{L})$ & Prof. $(\mathbf{m})$ & Temp. $\left({ }^{\circ} \mathrm{C}\right)$ & $\mathrm{pH}$ & Salinidad $(\mathbf{m g} / \mathbf{L})$ \\
\hline P1inv08 & 18,6 & 13,04 & 9910 & 0,32 & 1,6 & 8,55 & 11 \\
P2inv08 & 14,5 & 0,33 & 250 & 0,26 & 3,7 & 8,8 & 0,25 \\
P3inv08 & 13,6 & 4,53 & 3450 & 0,12 & 10,4 & 8,76 & 3,4 \\
P1ver09 & 8,5 & 2,75 & 2090 & 0,32 & 18,9 & 9,4 & 2 \\
P2ver09 & 8,7 & 0,21 & 170 & 0,45 & 14 & 8,8 & 0,15 \\
P3ver09 & 8,1 & 6,02 & 4570 & 0,18 & 25,4 & 9,5 & 4,56 \\
P1inv09 & 17 & 12,8 & 8552 & 0,31 & 12 & 8,5 & 10,88 \\
P2inv09 & 9,93 & 0,28 & 193 & 0,26 & 17 & 8,5 & 0,3 \\
PSCAT09 & 7,7 & 1,53 & 160 & 0,54 & 15,5 & 9,8 & 1,16 \\
P1ver10 & 11 & 4 & 1494 & 0,22 & 17 & 9 & 3 \\
P2ver10 & 9,6 & 0,09 & 67 & 0,27 & 16 & 7 & 0,06 \\
\hline
\end{tabular}

de la temperatura del agua, salinidad, oxígeno disuelto, profundidad y área del cuerpo de agua, sólidos totales y $\mathrm{pH}$; se agregaron dos variables ambientales "dummies" (instrumentales): invierno y temporada. Si la muestra fue colectada en invierno se le asignó el valor 1 y si provenía del verano 0 . En el caso de temporada (año de muestreo), se fijó 0 para 2009 y 1 para 2010. Estas variables con correlaciones mayores a 0,35 en cada eje de RDA fueron consideradas al momento de interpretar la importancia de las mismas en relación a las especies de diatomeas.

Se efectuaron biplots que grafican las especies y las puntuaciones ambientales de RDA. Por último, se realizaron análisis chi cuadrado para verificar si hay relación entre las temporadas y las estaciones del año con respecto a las abundancias de las especies de flamencos y de diatomeas en la laguna.

\section{Resultados}

En laguna de Los Pozuelos, durante diciembre de 2010 se verificó una importante reducción del espejo de agua de hasta un 99,73\%, cuando sólo conservó 28 ha. de las 10.600 alcanzadas en febrero de 2008. Dadas estas retracciones, durante invierno de 2009 sólo quedó agua en la zona sur y el Punto 3 se secó completamente. Además, en la laguna, la disponibilidad de agua para invierno de 2008 se vio reducida por la formación de una cubierta de hielo. Los cambios de superficie fueron acompañados por variaciones de la salinidad $\mathrm{y}$, de acuerdo a la clasificación de Cowardin et al. (1979) el cuerpo de agua osciló dentro de una condición oligo a mesosalina (3-11 g/L). Por su parte, los ríos estuvieron dentro del tipo oligosalina a dulce (0,06-1,16 g/L) lo que explica el marcado descenso de la salinidad en la laguna en el punto cercano a la desembocadura de los afluentes. En relación a las otras variables fisicoquímicas, las concentraciones de oxígeno disuelto descendieron en el verano; los valores de $\mathrm{pH}$ indicaron aguas alcalinas y la conductividad eléctrica alcanzó sus valores máximos en invierno (Tabla 1).

Dentro de las Bacillariophyceae se identificó un total de 36 géneros, con 116 taxones infragenéricos. Se destacaron Navicula y Nitzschia como los géneros mejor representados con 16 especies cada uno. La muestra con mayor riqueza específica fue para $\mathrm{P} 1$ en invierno de 2008, con 52 especies y la menor para el río Santa Catalina en verano de 2009, con 7 especies. Sólo para invierno de 2008 el registro de riqueza específica en la laguna fue mayor que la del Cincel (37 especies), mientras que en los otros períodos muestreados el Cincel llegó a superar en cinco veces el número de taxones (Tabla 2). En la laguna la riqueza específica fue disminuyendo notablemente desde el comienzo de las campañas (2008) hacia el final de las mismas (2010), con registros extremos durante el invierno ( $8-52$ especies), mientras que en el río Cincel el número de especies se mantuvo más 
Bol. Soc. Argent. Bot. 49 (2) 2014

Tabla 2. Distribución de taxones de diatomeas en los puntos muestreados. P1 y P3 (laguna); P2 (río Cincel), SCat (río Santa Catalina); I: invierno; V: verano.

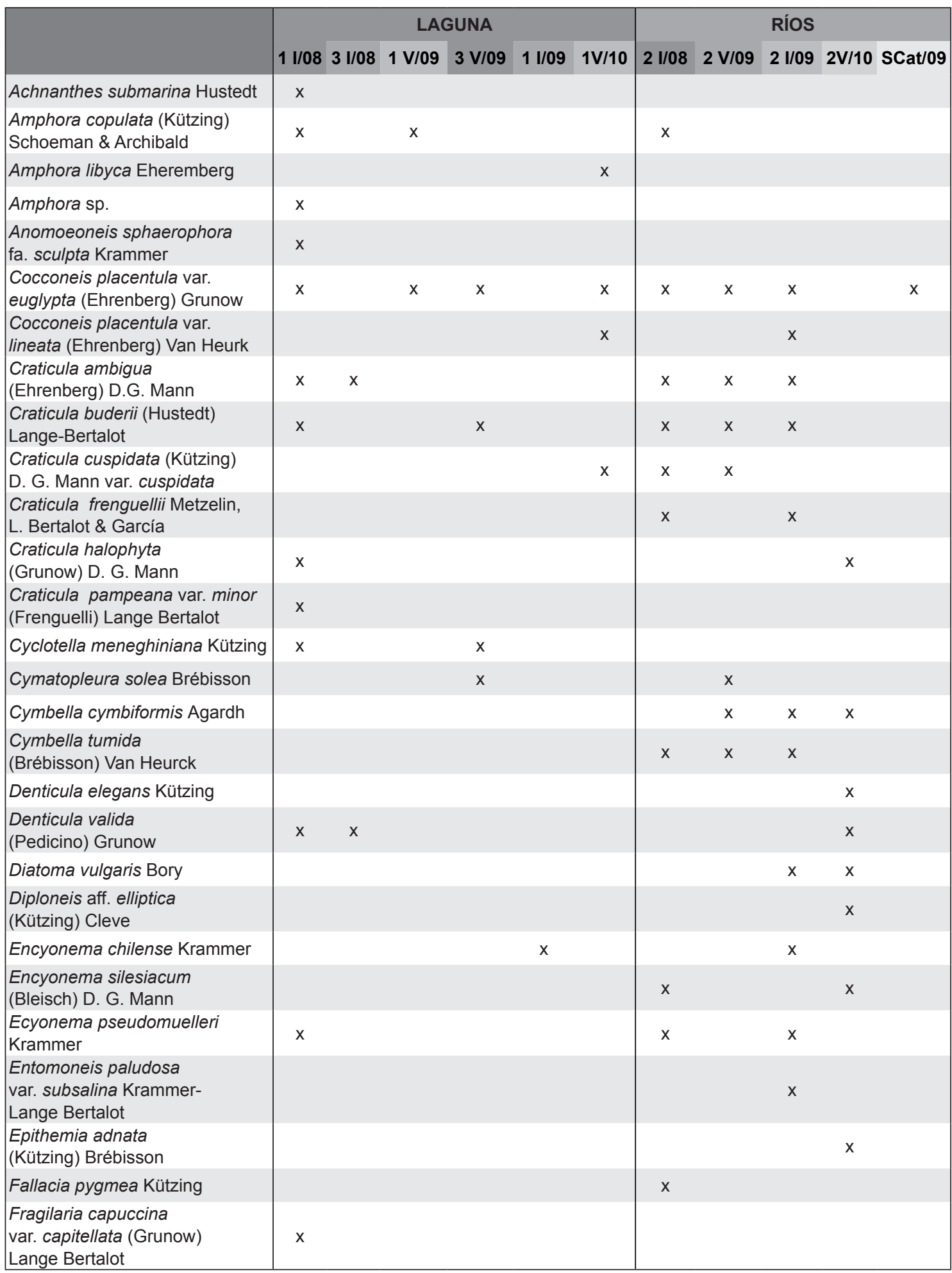


A. L. González Achem et al. - Flora diatomológica en Laguna de Los Pozuelos

\begin{tabular}{|c|c|c|c|c|c|c|c|c|c|c|c|}
\hline & \multicolumn{6}{|c|}{ LAGUNA } & \multicolumn{5}{|c|}{ Ríos } \\
\hline & $1 \mathrm{l} / 08$ & $31 / 08$ & $1 \mathrm{~V} / 09$ & $3 \mathrm{~V} / 09$ & $1 \mathrm{l} / 09$ & $1 \mathrm{~V} / 10$ & $21 / 08$ & 2 V/09 & $21 / 09$ & $2 \mathrm{~V} / 10$ & SCat/09 \\
\hline $\begin{array}{l}\text { Fragilaria capuccina var. } \\
\text { vaucheriae (Kützing) } \\
\text { Lange-Bertalot }\end{array}$ & $x$ & & & $x$ & & & $\mathrm{x}$ & $x$ & $\mathrm{x}$ & $x$ & $x$ \\
\hline $\begin{array}{l}\text { Gomphonema anglicum } \\
\text { Eherenberg }\end{array}$ & $x$ & & & & & & & & & & \\
\hline $\begin{array}{l}\text { Gomphonema capitatum } \\
\text { Ehrenberg }\end{array}$ & & & & & & & & $x$ & $x$ & $x$ & \\
\hline $\begin{array}{l}\text { Gomphonema parvulum } \\
\text { (Kützing) Kützing }\end{array}$ & $\mathrm{x}$ & $x$ & & $x$ & & & $x$ & $x$ & $x$ & $x$ & \\
\hline $\begin{array}{l}\text { Gomphonema aff. } \\
\text { gracile Ehrenberg }\end{array}$ & & & & & & & & $x$ & & & \\
\hline Gomphonema sp. 1 & $x$ & & & & & & & $x$ & $x$ & & \\
\hline Gomphonema sp. 2 & & & & & & & & $x$ & $x$ & & \\
\hline $\begin{array}{l}\text { Gyrosigma acuminatum } \\
\text { (Kützing) Rabenhorst }\end{array}$ & $\mathrm{x}$ & & & & & $x$ & $\mathrm{x}$ & $x$ & $x$ & & $x$ \\
\hline Gyrosigma sp. 1 & & & & & & & $x$ & & & & \\
\hline Gyrosigma sp. 2 & & & & & & & & & & & $x$ \\
\hline $\begin{array}{l}\text { Halamphora coffaeformis } \\
\text { (Agardh) Kützing }\end{array}$ & & & & $x$ & & & & & & & \\
\hline $\begin{array}{l}\text { Halamphora veneta } \\
\text { (Kützing) Levkov }\end{array}$ & $\mathrm{x}$ & $x$ & $x$ & $x$ & & $x$ & $x$ & $x$ & $x$ & $x$ & $x$ \\
\hline $\begin{array}{l}\text { Halamphora aff. montana } \\
\text { (Krasske) Levkov }\end{array}$ & $x$ & & & & & & & & & & \\
\hline $\begin{array}{l}\text { Hantzschia amphioxis } \\
\text { (Ehrenberg) Grunow }\end{array}$ & $\mathrm{x}$ & & & & & & $x$ & $x$ & $x$ & $x$ & $x$ \\
\hline Hipodonta capitata Patrick & & $x$ & & $x$ & & $x$ & $x$ & $x$ & $x$ & & \\
\hline $\begin{array}{l}\text { Hipodonta hungarica } \\
\text { (Grunow) Lange-Bertalot }\end{array}$ & $x$ & $x$ & $x$ & $x$ & & & $x$ & $x$ & $x$ & & \\
\hline $\begin{array}{l}\text { Luticola cohnii (Hilse) } \\
\text { Lange-Bertalot }\end{array}$ & $x$ & & & & $x$ & & & & & & \\
\hline $\begin{array}{l}\text { Luticola molis Lange- } \\
\text { Bertalot \& Rumrich }\end{array}$ & $x$ & & & & & & & & & & \\
\hline $\begin{array}{l}\text { Luticola nivalis (Ehrenberg) } \\
\text { D.G. Mann }\end{array}$ & $x$ & & & & & & & & & & \\
\hline Luticola sp. & & & & & & & & & & $x$ & \\
\hline Melosira varians Agardh & & & & & & & & & $x$ & & \\
\hline $\begin{array}{l}\text { Navicula broetzii Lange } \\
\text { Bertalot \& Reichardt }\end{array}$ & & & $x$ & & & & & & & & \\
\hline $\begin{array}{l}\text { Navicula capitatoradiata } \\
\text { Germain }\end{array}$ & & $x$ & & & & & & & $\mathrm{x}$ & & \\
\hline Navicula cincta Ehrenberg & $x$ & $x$ & & $x$ & $x$ & & & $x$ & & & \\
\hline Navicula cryptocephala Kützing & & $x$ & & $x$ & & & & $x$ & $x$ & $x$ & \\
\hline $\begin{array}{l}\text { Navicula lauca Rumrich } \\
\text { \& Lange-Bertalot }\end{array}$ & & $x$ & & & & $x$ & & & & & \\
\hline Navicula libonensis Schoemann & $x$ & & & & & & & $x$ & & & \\
\hline $\begin{array}{l}\text { Navicula parinacota Rumrich } \\
\text { \& Lange-Bertalot }\end{array}$ & & $x$ & & $x$ & & & $x$ & & & & \\
\hline $\begin{array}{l}\text { Navicula peregrina } \\
\text { (Ehrenberg) Kützing }\end{array}$ & & & & & & $x$ & & & & & \\
\hline Navicula pseudogracilis Hustedt & & $x$ & & & & & & & & & \\
\hline $\begin{array}{l}\text { Navicula salinicola } \\
\text { Hustedt var. salinicola }\end{array}$ & & & $\mathrm{x}$ & $x$ & & & $\mathrm{x}$ & & & & \\
\hline
\end{tabular}


Bol. Soc. Argent. Bot. 49 (2) 2014

\begin{tabular}{|c|c|c|c|c|c|c|c|c|c|c|}
\hline & \multicolumn{6}{|c|}{ LAGUNA } & \multicolumn{4}{|c|}{ Ríos } \\
\hline & $1 \mathrm{l} / 08$ & $31 / 08$ & $1 \mathrm{~V} / 09$ & $3 \mathrm{~V} / 09$ & $1 \mathrm{l} / 09$ & $1 \mathrm{~V} / 10$ & $21 / 08$ & $2 \mathrm{~V} / 09$ & $21 / 09$ & 2V/10 SCat/09 \\
\hline Navicula trivialis Lange Bertalot & & & & $x$ & & & & & & \\
\hline Navicula veneta Kützing & $x$ & $x$ & & $x$ & & $x$ & & & & \\
\hline $\begin{array}{l}\text { Navicula aff. erifuga } \\
\text { Lange Bertalot }\end{array}$ & & $x$ & & & & & & $x$ & & \\
\hline $\begin{array}{l}\text { Navicula aff. lohmani } \\
\text { Lange- Bertalot \& Rumrich }\end{array}$ & & & & $x$ & & & & & & \\
\hline Navicula sp. 4 Lange-Bertalot & & & & $\mathrm{x}$ & & & & & & \\
\hline Navicula sp. & & $x$ & $x$ & & & & & & $x$ & \\
\hline Nitzschia austriaca Hustedt & $x$ & & & & & & & & & \\
\hline Nitzschia bacillum Hustedt & $x$ & & & & & & & & & \\
\hline Nitzschia calida Grunow & $x$ & & & & & & & $x$ & & \\
\hline $\begin{array}{l}\text { Nitzschia compresa } \\
\text { (Bailey) Boyer }\end{array}$ & & $x$ & & $x$ & & & & & & \\
\hline $\begin{array}{l}\text { Nitzschia frustulum var. } \\
\text { subsalina Hustedt }\end{array}$ & $\mathrm{x}$ & & & & & & & & & \\
\hline $\begin{array}{l}\text { Nitzschia halloyi } \\
\text { Maidana \& Herbst }\end{array}$ & & & & & & & & $x$ & & \\
\hline Nitzschia insconspicua Grunow & $x$ & & & & & & & & & \\
\hline Nitzschia intermedia Hantzsch & $x$ & & & & & & $x$ & & & \\
\hline $\begin{array}{l}\text { Nitzschia palea } \\
\text { (Kützing) W. Smith }\end{array}$ & $x$ & & & $x$ & & & $x$ & $x$ & $x$ & $x$ \\
\hline $\begin{array}{l}\text { Nitzschia sigma } \\
\text { (Kützing) W. Smith }\end{array}$ & $\mathrm{x}$ & $x$ & & $x$ & & & $x$ & $x$ & $x$ & \\
\hline Nitzschia solita Hustedt & $x$ & $x$ & & & & & & & & \\
\hline $\begin{array}{l}\text { Nitzschia supralitorea } \\
\text { Lange-Bertalot }\end{array}$ & $\mathrm{x}$ & & & & & & & & & \\
\hline Nitzschia tryblionella Hantzsch & $x$ & $x$ & & & $x$ & $x$ & & & $x$ & \\
\hline $\begin{array}{l}\text { Nitzschia valdecostata Lange- } \\
\text { Bertalot \& Simonsen }\end{array}$ & $x$ & $x$ & & & & & & & & \\
\hline $\begin{array}{l}\text { Nitzschia aff. subacicularis } \\
\text { Hustedt }\end{array}$ & & & & & & & & $x$ & & $x$ \\
\hline Pinnularia borealis Ehrenberg & & & & $x$ & & & & & $x$ & $x$ \\
\hline $\begin{array}{l}\text { Pinnularia borealis var. } \\
\text { undulata Hustedt }\end{array}$ & & & & & & & & & & $x$ \\
\hline Pinnularia divergens W. Smith & & & & & & & & $x$ & & \\
\hline $\begin{array}{l}\text { Pinnularia maior (Kützing) } \\
\text { Rabhenhorst }\end{array}$ & & & & & & & & & & $x$ \\
\hline $\begin{array}{l}\text { Pinnularia microstauron } \\
\text { (Eherenberg) Cleve }\end{array}$ & $\mathrm{x}$ & $x$ & & & & & & $x$ & $\mathrm{x}$ & $x$ \\
\hline $\begin{array}{l}\text { Pinnularia viridis } \\
\text { (Nitzsche) Ehrenberg }\end{array}$ & $\mathrm{x}$ & & & & & & $\mathrm{x}$ & $x$ & & \\
\hline Placoneis sp. & & & & & & & & $\mathrm{x}$ & & \\
\hline Planothidium delicatulum Kützing & $x$ & $x$ & $x$ & $x$ & $x$ & $x$ & $x$ & $x$ & $x$ & $x$ \\
\hline $\begin{array}{l}\text { Planothidium frequentissimum } \\
\text { (Lange-Bertalot) Lange-Bertalot }\end{array}$ & & & & & & & $x$ & & & \\
\hline $\begin{array}{l}\text { Planothidium lanceolatum } \\
\text { (Brébisson ex Kützing) } \\
\text { Lange Bertalot }\end{array}$ & $\mathrm{x}$ & & & & & $x$ & $x$ & & & $x$ \\
\hline $\begin{array}{l}\text { Planothidium aff. engelbrechti } \\
\text { (Cholnoky) Maidana }\end{array}$ & & & & & & $x$ & $x$ & & & $x$ \\
\hline
\end{tabular}


A. L. González Achem et al. - Flora diatomológica en Laguna de Los Pozuelos

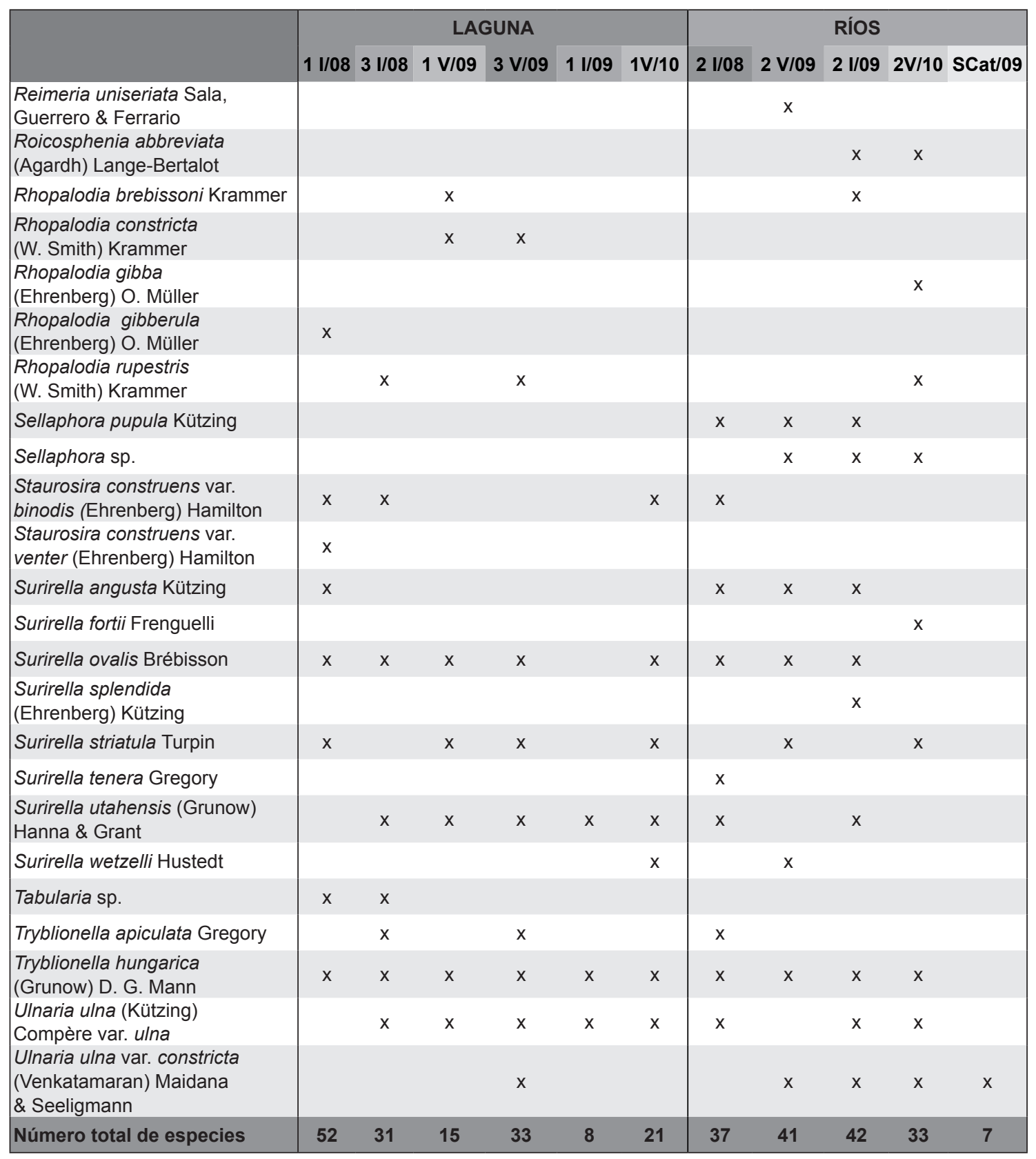

constante. El número total de especies exclusivas para cada uno de los ambientes (laguna y río) fue similar, con 34 y 32 taxones respectivamente. Para invierno se registraron 35 especies exclusivas, mientras que 22 fueron observadas únicamente en verano.

Las especies que se encontraron con una frecuencia de ocurrencia mayor al $50 \%$ del total de muestras colectadas fueron 18 (Cocconeis placentula var. euglypta, Craticula ambigua, Fragilaria capuccina var. vaucheriae, Gomphonema parvulum, Gyrosigma acuminatum, Halamphora veneta, Hantzschia amphioxis, Hipodonta capitata, H. hungarica, N. palea, N. sigma, Planothidium delicatulum, P. lanceolatum, Surirella ovalis, S. utahensis, S. wetzelli, Tryblionella hungarica y Ulnaria ulna) y el porcentaje de especies poco frecuentes (observadas una sola vez) fue elevado (40\%). De las 18 especies 


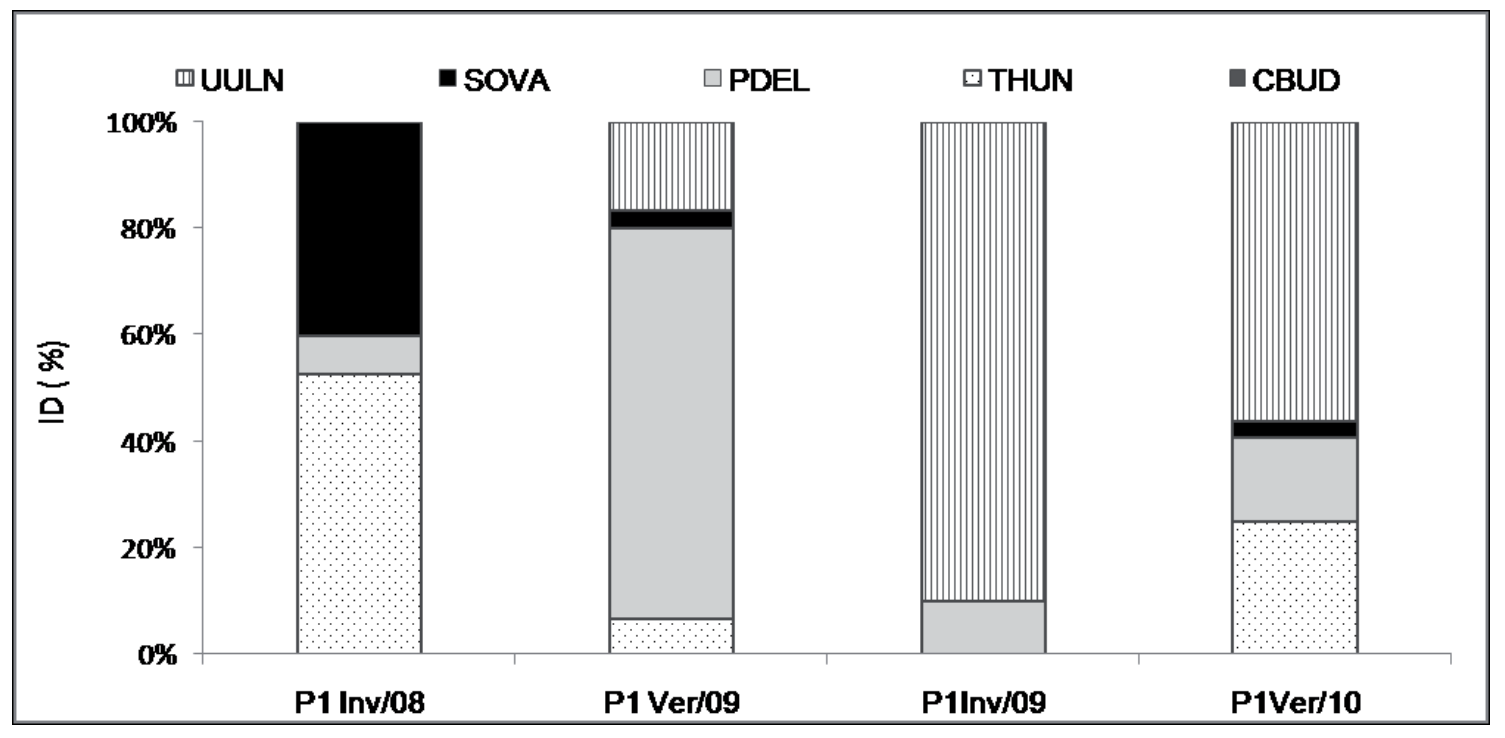

Fig. 2. Niveles de dominancia porcentual (ID \%) de las especies de diatomeas registradas en la laguna de Los Pozuelos (P1) durante los veranos (ver) de 2009 y 2010 e inviernos (inv) de 2008 y 2009.

mencionadas, 14 tuvieron abundancia relativa superior al $5 \%$ en algún muestreo y para sólo 5 especies fue mayor al $20 \%$ (Tabla 2). Los mayores registros de diversidad $(\mathrm{H})$ se obtuvieron para el río Cincel en verano $(4,55)$ y para la laguna durante invierno $(3,74)$.

La oferta de diatomeas fue variable en relación a las estaciones. Los valores máximos de abundancia en la laguna correspondieron tanto para invierno (Julio/2008) como para verano (Febrero/2010), en los momentos de los mínimos registros de superficie (9.767 y 8.389 ha, respectivamente). Sin embargo, se detectaron diferencias en las abundancias relativas entre las estaciones húmedas (verano) y secas (invierno) y entre los años de muestreo. Para la laguna (Fig. 2), en invierno de 2008, S. ovalis junto a T. hungarica fueron dominantes, mientras que para esta estación en 2009 se destacó U. ulna. En el primer verano muestreado (2009) resaltó por su abundancia P. delicatulum y para 2010 esta especie estuvo junto a T. hungarica y $U$. ulna aunque esta última presentó mayores abundancias. En el río Cincel (Fig. 3), se observó una fuerte dominancia de especies sólo en el verano de 2010 con $H$. amphioxis, U. ulna y en tercer lugar a $P$. delicatulum. Durante la época seca el aporte de diatomeas fue realizado principalmente por U. ulna, P. delicatulum, T. hungarica, N. palea y C. buderi y en el verano por U. ulna, H. amphioxis,
P. delicatulum, T. hungarica y $S$. ovalis. Aunque $P$. delicatulum estuvo presente tanto en estación seca como húmeda de ambas temporadas, se destaca las bajas abundancias de la misma en el invierno de 2008 y, la ausencia total de $N$. palea en verano de 2010.

En relación al tamaño, en la laguna, durante el invierno, las especies con mayor abundancia relativa fueron las de mayor dimensión (65 y $148 \mu \mathrm{m})$ y en el verano las más pequeñas como $P$. delicatulum (18-23 $\mu \mathrm{m}$ de largo), aunque también estuvo presente U. ulna con 73-207 $\mu \mathrm{m}$ de largo (Fig. 2). En el río Cincel, en invierno fueron más abundantes las especies de menor tamaño como C. buderi (23$32 \mu \mathrm{m})$ у $P$. delicatulum o algo más grandes como $U$. ulna y T. hungarica $(40-65 \mu \mathrm{m})$ y se observó una mayor diversidad de organismos (Fig. 3). En este río los dos veranos se comportaron diferentes: para 2009 no se destacó ninguna especie, mientras que en 2010 sobresalieron por su abundancia U. ulna y H. amphyoxis $(35-81 \mu \mathrm{m})$.

Las abundancias totales de las tres especies de flamencos y diatomeas en la laguna resultaron estar asociadas significativamente $(\mathrm{p}<0.05)$ entre invierno y verano y los años de muestreo $\left(\chi^{2}=240,75\right.$ para $P$. andinus; $\chi^{2}=52,81$ para $P$. jamesi $; \chi^{2}=240,07$ para $P$. chilensis y $\chi^{2}=134.48$ para diatomeas). En el caso de los flamencos la abundancia en verano 


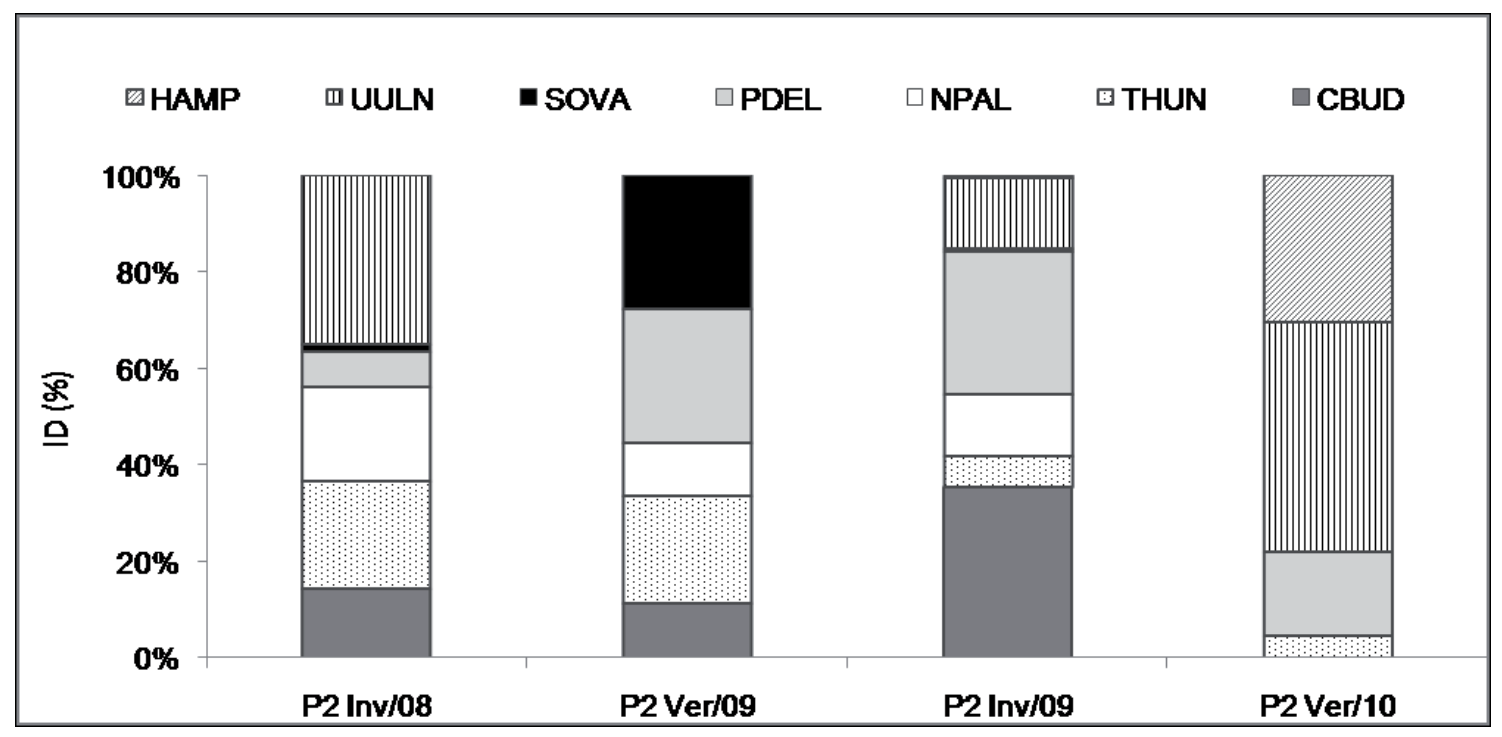

Fig. 3. Niveles de dominancia porcentual (ID \%) de las especies de diatomeas registradas en el río Cíncel (P2) durante los veranos (ver) de 2009 y 2010 e inviernos (inv) de 2008 y 2009.

de 2010 fue significativamente mayor $(\mathrm{N}=21.843$ individuos) que verano de $2009(\mathrm{~N}=9.339)$ y el menor registro le correspondió a Julio de 2008 (N= 3.139 individuos). La especie más abundante de flamencos fue $P$. chilensis. P. jamesi estuvo siempre presente, aunque en menor número durante el verano (Fig. 4).

En la figura 5 se observa el ordenamiento de las muestras en el análisis multivariado NMDS (STRESS $=0.0003, \mathrm{p}<0.05$ ). El análisis de correlación de Spearman entre cada eje extraído de ordenamiento y las variables ambientales utilizadas mostró una correlación positiva significativa entre el eje 1 y la diversidad $\left(r_{s p}=0,68, p<0.05\right)$ y correlaciones negativas significativas entre este mismo eje y sólidos totales $\left(\mathrm{r}_{\mathrm{sp}}=-0,98, \mathrm{p}<\right.$ $0.05)$ y salinidad $\left(\mathrm{r}_{\mathrm{sp}}=-0,97, \mathrm{p}<0.05\right)$. Los resultados indicaron que el $\mathrm{P} 2$ tuvo características propias distintivas de los otros sitios (P1 y P3), e independientes de las estaciones del año, con mayores valores de diversidad y menores valores de salinidad y sólidos totales. En invierno, P1 obtuvo los mayores valores de salinidad y sólidos totales mientras que en verano de 2010 , este sitio se comportó como P2.

Los resultados del RDA (Fig. 6 A-C) mostraron que los tres primeros ejes canónicos acumularon el $79 \%$ de la varianza total, con un $41 \%$ representado en el primer eje, un $26 \%$ para el segundo y un $12 \%$ para el tercer eje (inercia $=0.043, \mathrm{p}<0.05)$.

El eje 1 (Fig. 6 A) sintetizó la distribución de las diatomeas principalmente a lo largo de un gradiente negativo de salinidad (corr $=-0.55$ ) y positivo de profundidad (corr $=0.39$ ). Este eje permite distinguir dos grupos de especies: (I) formado por C. buderi, F. capuccina var. capitellata, G. parvulum, N. palea y U. ulna var. constricta que estuvo, en general, mejor representado en los ríos o en la laguna en condiciones de mayor profundidad y menor salinidad. El segundo grupo (II) estuvo integrado por las especies $S$. construens var. binodis, $S$. utahensis y $U$. ulna que fueron más abundantes en la laguna cuando los registros de salinidad aumentaron y la profundidad disminuyó.

El eje 2 (Fig. 6 B) se correlacionó positivamente con el $\mathrm{pH}($ corr $=0.47)$. Las mayores abundancias de las especies $C$. placentula var. euglypta, T. hungarica y $S$. ovalis estuvieron asociadas con aguas alcalinas y las de $F$. capuccina var. capitellata, N. palea, U. ulna y U. ulna var. constricta, con registros de $\mathrm{pH}$ más bajo.

El eje 3 (Fig. 6 C) que sintetiza un efecto estacional, se correlacionó positivamente con la temperatura (corr $=0.48)$ y con la temporada (corr $=0.79)$, pero negativamente con OD $($ corr $=-0.51)$, con la estación invierno (corr $=-0.80)$ y el área 
Bol. Soc. Argent. Bot. 49 (2) 2014

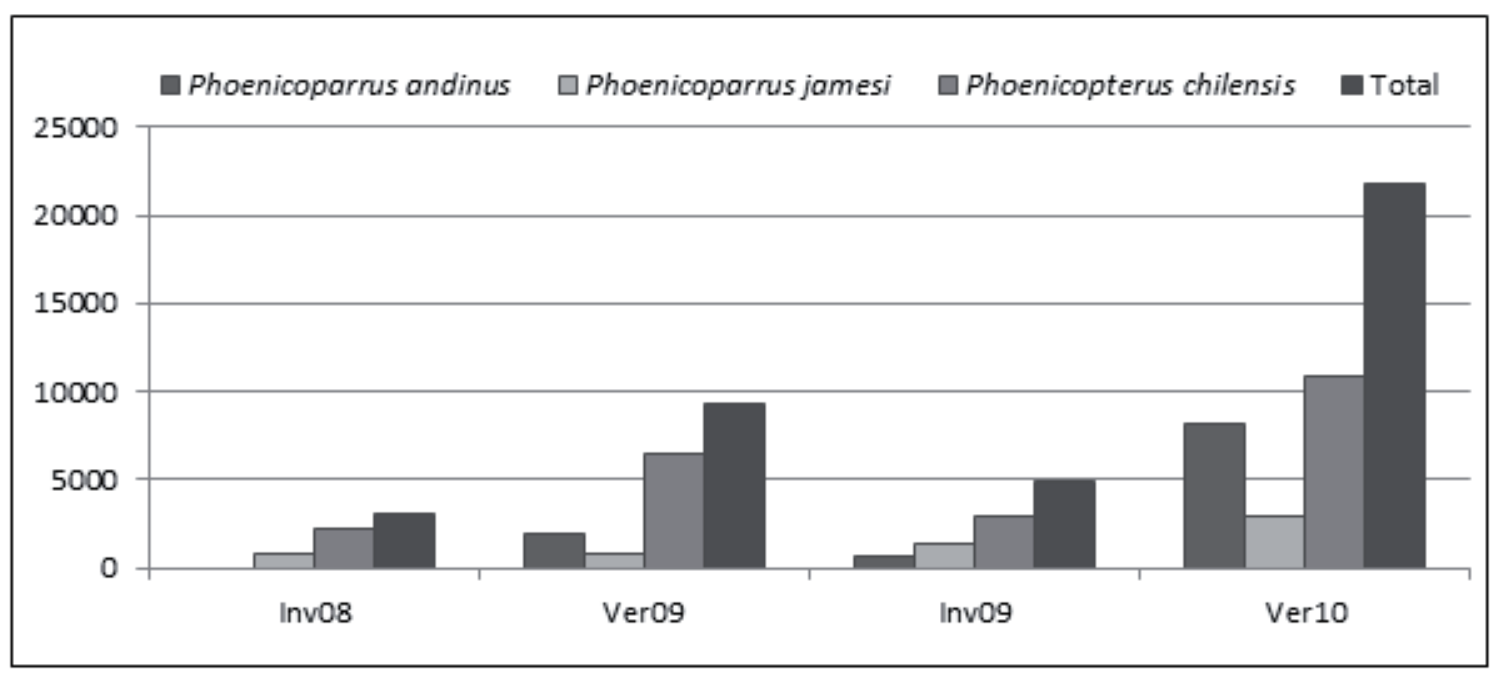

Fig. 4. Abundancias de las tres especies de flamencos y totales para la Laguna de Los Pozuelos.

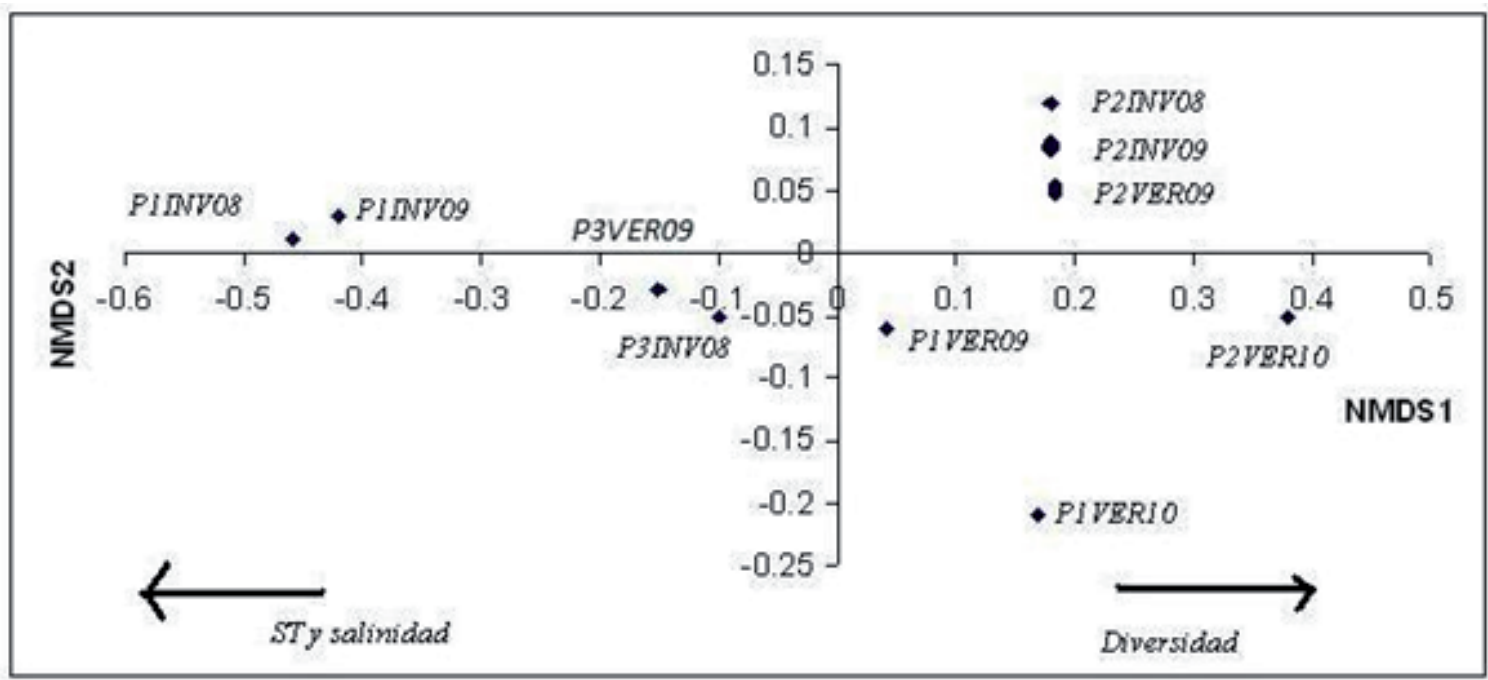

Fig. 5. Ordenamiento de las muestras según el NMDS basado en variables ambientales. Se consignan las variables que mostraron una correlación no paramétrica significativa con los ejes extraídos.

(corr $=-0.78)$. Las mayores abundancias de $C$. placentula var. euglypta y $H$. veneta se registraron bajo condiciones temperaturas más elevadas, bajos valores de OD, cuando la laguna tuvo menos superficie, durante verano de 2010. Por su parte, las mayores abundancias de G. parvulum, C. buderi y $H$. hungarica ocurrieron a bajas temperaturas, mayor superficie y concentración de oxígeno disuelto durante invierno de 2008.

\section{Discusión y Conclusiones}

Las variaciones en el área de la laguna debidas a la estacionalidad del régimen hidrológico de los tributarios, condicionaron las fluctuaciones físico-químicas y composición de la comunidad de diatomeas.

Como cabe esperar en lagos salinos (Hammer, 1978), la concentración de sales fue mayor durante 


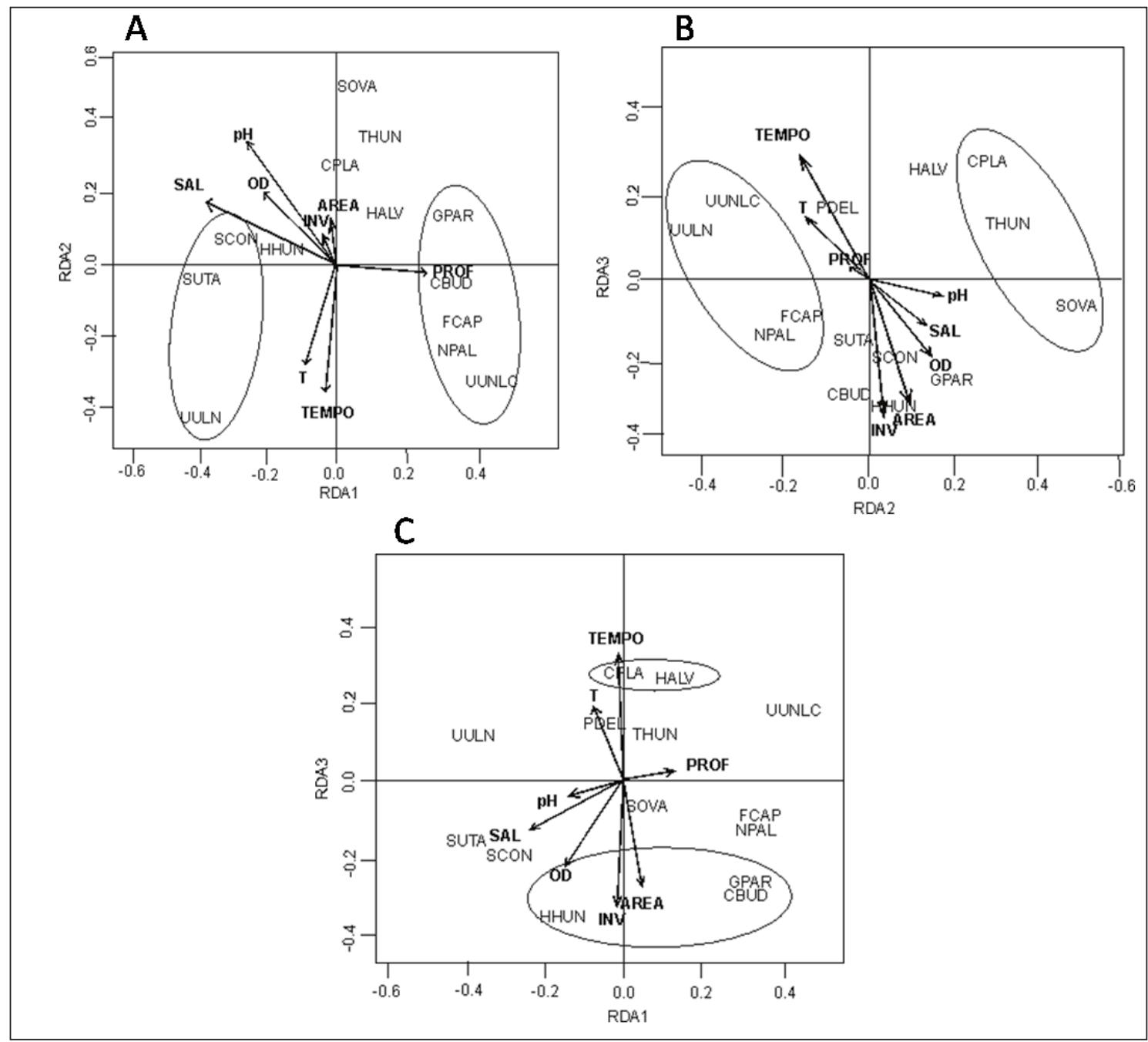

Fig. 6 A-C. Distribución de las especies de diatomeas en un diagrama de ordenación con los ejes RDA1, RDA2 y RDA3 del Análisis de Redundancia (RDA). Las variables ambientales están representadas por flechas que indican la dirección en la cual las variables incrementan. Se representan los taxones de diatomeas como sigue: CPLA (Cocconeisplacentula var. euglypta), CBUD (Craticula buderi), FCAP (Fragilaria capuccina var. capitellata), GPAR (Gomphonema parvulum), HALV (Halamphora veneta), HHUN (Hipodonta hungarica), NPAL (N. palea), PDEL (Planothidium delicatulum), SCON (Staurosira construens var. binodis), SOVA (Surirella ovalis), SUTA (S. utahensis), THUN (Tryblionella hungarica), UULN (Ulnaria ulna), UULNC: (U. ulna var. constricta). OD (oxígeno disuelto), PH (pH), SAL (Salinidad), INV (Invierno), PROF (Profundidad), T (Temperatura), AREA, TEMPO (Temporada).

los meses en los cuales la evaporación excedió a las precipitaciones (invierno). La composición iónica de Laguna de Los Pozuelos, dominada por el sodio sobre los otros cationes, es también una secuencia común en lagos salinos de Asia, Australia y Norte y Sud América (Campos et al., 1996).
Iltis et al. (1984) y Blinn (2001) entre otros autores, hallaron una correlación negativa entre la diversidad y la riqueza de especies con la salinidad, lo que no pudo ser verificado en Pozuelos. En general, en el río Cincel la riqueza específica fue mayor que en la laguna, lo que concordaría con el patrón descripto. Sin embargo, en la laguna 
Pozuelos, en invierno 2008 se obtuvieron los registros más elevados tanto de salinidad como de riqueza.

La mayoría de las especies encontradas son bentónicas y muchas de ellas, como por ejemplo Denticula elegans, Hantzschia amphioxis, Nitzschia palea, N. supralitorea y Pinnularia borealis, son capaces de vivir en ambientes someros, sometidos a desecaciones (Van Dam et al., 1994).

Alrededor de catorce especies características de ambientes alcalinos, ligeramente salobres a salobres (A. lybica, Cocconeis placentula var. euglypta, Craticula cuspidata var. cuspidata, C. halophyla, H. amphyoxis, Navicula cincta, Nitzschia frustulum var. subsalina, N. sigma, Planothidium delicatulum, P. lanceolatum, Sellaphora pupula, Surirella striatula, T. hungarica y T. apiculata) (Krammer \& Lange-Bertalot, 1986, 1988, Servant-Vildary \& Mello e Sousa, 1993 y Licursi et al., 2010), obtuvieron en alguna muestra una frecuencia de ocurrencia superior al $50 \%$. Ninguna especie estuvo presente en todas las muestras y sólo tres fueron halladas en 10 de las 11 recolectadas.

La abundancia de diatomeas en la laguna fluctuó según la temporada húmeda (verano) o seca (invierno), como lo señalaron Caziani \& Derlindati (2000), Masciti (1996) y Maidana et al. (1998). Los diferentes resultados obtenidos para una misma estación del año estuvieron en estrecha relación con el volumen de agua que tuvo la laguna. Las figuras 2 y 3 , relacionadas con el índice de dominancia de las especies de diatomeas para laguna y río respectivamente, sugieren que éste tuvo mayor equitatividad (menor efecto de dominancia) respecto a la laguna en la distribución de las especies, en todas las temporadas. Esto indicaría menor estabilidad en la comunidad de diatomeas en el ambiente léntico como resultado de las contracciones periódicas a las que se encuentra sometida la laguna en respuesta a los cambios ambientales.

Por otro lado, es interesante considerar que la disminución de la abundancia de las diatomeas observada a partir de febrero de 2009 pudo deberse a la fuerte presión de pastoreo ejercida por una bandada grande de flamencos ocurrida en julio de 2008 y cuyo efecto persistió durante varios meses como lo ocurrido en la laguna Puripica Chico, (Bolivia) (Hulbert \& Chang, 1983).
El análisis NMDS confirmó que el río Cincel tuvo un comportamiento estable, tanto estacionalmente como en los diferentes años de muestreo y que los puntos de muestreo correspondientes a la laguna (P1 y P3) cambiaron sus condiciones de acuerdo a la estación del año.

El análisis de RDA sugiere la diferenciación de tres grupos principales de especies: 1.- Tolerantes a la salinidad y bajas temperaturas como $S$. construens var. binodis, S. utahensis y U. ulna, propias de la laguna; 2.- Más abundantes en el río, en condiciones de baja salinidad y temperatura: C. buderi, F. capuccina, G. parvulum, N. palea y U. ulna var. constricta; 3.- Especies alcalófilas formado por $C$. placentula var. euglypta, T. hungarica y $S$. ovalis.

Nuestros resultados difieren de los señalados por otros autores sólo para $S$. construens var. binodis, y U. ulna, ya que estas especies fueron reportadas para aguas de tipo dulce a oligosalinas (De Wolf, 1982; Blinn, 2001).

T. hungarica, que se desarrolló especialmente en el verano, fue registrada con abundancias relativamente altas también en invierno de 2008 para el sitio P1. Esta especie fue reportada para un amplio rango de salinidad desde dulce a mesosalina (Maidana et al. 2011).

Los resultados obtenidos demostraron que las grandes variaciones a las que están sometidos los cuerpos de agua de altura limitan las posibles predicciones que se puedan hacer sobre su comportamiento, por lo que es imprescindible efectuar seguimientos sistemáticos en este tipo de ambientes.

No se pudo identificar ningún patrón que permitiera interpretar las variaciones ocurridas en la abundancia de los flamencos y que estuviera relacionado con la disponibilidad de las diatomeas. La ausencia de $P$. andinus para invierno de 2008 no estaría explicada por la falta de alimento ya que durante esta estación se registró la mayor diversidad de presas, con diatomeas de tamaño adecuado para esta ave. Además, P. jamesi que ingiere presas pequeñas, estuvo en tercer lugar en abundancia durante los veranos, cuando predominaron diatomeas de menor tamaño.

Se registraron por primera vez para la laguna 30 taxones (Achnanthes submarina, Craticula ambigua, C. buderi, C. frenguellii, C. pampeana var. minor, Cymatopleura solea, Cymbella cymbiformis, Denticula valida, Diatoma vulgaris, Encyonema 


\section{A. L. González Achem et al. - Flora diatomológica en Laguna de Los Pozuelos}

chilense, E. pseudomuelleri, Epithemia adnata, Gomphonema anglicum, Luticola mollis, Navicula broetzii, $N$. cincta, N. lauca, N. parinacota, $N$. pseudogracilis, Nitzschia calida, N. frustulum var. subsalina, $N$. intermedia, Pinnularia borealis var. undulata, Reimeria uniseriata, Rhopalodia rupestris, Staurosira construens var. binodis, Surirella fortii, S. splendida, S. wetzelli, Tryblionella apiculata y Ulnaria ulna var. constricta), mientras que de estas 18 son nuevas citas para la provincia de Jujuy.

\section{Agradecimientos}

Expresamos nuestro agradecimiento a la Delegación Regional Noroeste de Parques Nacionales para el Sistema de Información de Biodiversidad por habernos brindado la oportunidad de trabajar en estos bellos lugares.

\section{Biblografía}

ARAGÓN, R. \& J. M. MORALES. 2003. Species composition and invasion in NW Argentinian secondary forests: effects of land use history, environment and landscape. J. Veg. Sci.14: 195-204.

BATTARBEE, E. W. 1986. Diatom Analysis. In: BERGLUND, B. E. (ed.), Handbook of Holocene Palaeoecology and Palaeohydrology, pp. 527-570. J. Wiley \& Sons Ltd., New York.

BLINN, D. W. 2001. Diatom community structure along physicochemical gradients in Saline lakes. Ecology 74: 1246-1263.

CABRERA, A. \& A. WILLINK. 1973. Biogeografía de América Latina. Programa regional de desarrollo científico y tecnológico. Secretaría General de la Organización de los Estados Americanos, Washington D.C.

CAJAL, J. L. 1998. Las unidades morfoestructurales, el clima, la vegetación y las poblaciones humanas en la Puna y Cordillera Frontal. In: CAJAL, J. L., J. GARCÍA FERNÁNDEZ \& R. TECCHI (eds.), Bases para la conservación y manejo de la Puna y Cordillera Frontal de Argentina. El rol de las reservas de biosfera, pp. 1-24. FUCEMA, UNESCO, Uruguay.

CAMACHO, M. 2009. Sedimentología y mineralogía de los depósitos superficiales aluviales y lacustres del bolsón de la Laguna de los Pozuelos, (BLLP), Puna Jujeña, Argentina. Tesis Doctoral $N^{\circ} 57$, Universidad Nacional de Tucumán.
CAMPOS, H, S. SOTO, O. PARRA, W. STEFFEN \& G. AGÜERO. 1996. Limnological studies of Amarga Lagoon, Chile: a saline lake in Patagonian South America. Int. Jour. Salt Lake Res. 4: 301-3014.

CAZIANI, S. \& E. DERLINDATI. 2000. Abundance and habitat of Andes flamingos in northwestern Argentina. Waterbirds 23: 121-133.

COWARDIN, L. M., V. CARTER, F. C. GOLET \& E.T. LAROE. 1979. Classification of wetlands and deep water habitats of the United States. U. S. Department of the Interior, Fish and Wildlife Service, Washington, D.C.

DEJOUX, C. 1993. Benthic invertebrates of some lakes of Sud Lipez región, Bolivia. Hydrobiologia 267: 257-267.

DE WOLF, H. 1982. Method of coding of ecological data from diatoms for computer utilization. Mededel. Rijks Geol. Dienst. 36: 95-110.

DÍAZ, C. \& N. I. MAIDANA. 2005. Diatomeas de los salares de Atacama y Punta Negra II región. Chile. Centro de Ecología Aplicada.

FRENGUELLI, J. 1928. Acerca del origen de los salares de la región de los desiertos de la Puna de Atacama. GAEA. Anal. Soc. Est. Geogr. 3: 167-186.

GARCÍA FERNÁNDEZ, J. \& Y R. TECCHI. 1991. La Reserva de la Biósfera Laguna de los Pozuelos: un ecosistema pastoril en los Andes centrales. PERINBIAL UNJu, Argentina, Unesco ORCYT-MAB.

HAMMER, U. T. 1978. The saline lake of Saskatchewan. III. Chemical characterization. Inter. Rev. Gesam. Hydrobiol. 63: 311-335.

HURLBERT, S. H. 1982. Limnological studies of flamingo investigations and distributions. Nat. Geo. Res. Rep. 14: 351-356.

HURLBERT, S. H. \& C. C CHANG. 1983. Ornitholimnology: effects of grazing by the Andean flamingo (Phoenicoparrus andinus). Proc. Natl. Acad. 80: 4766-4769.

HUSTEDT, F. 1927. Fossile Bacillariaceen aus dem Loa -Becken in der Atacama-Wuste, Chile. Arch. Hydrobiol. 18: 224-251.

HUSTEDT, F. 1930. Bacillariophyta (Diatomae). In: PASCHER, A. (ed.), Die Süsswasser-Flora Mitteleuropas, pp. 1- 466. Gustav Fischer, Jena.

HUSTEDT, F. 1959-1966. Die Kieselalgen. In: RABENHORST, L. (ed.), Kryptogamen-flora von Deutschland, Osterreich und der Schweiz, 7 (2) 6: 737-845 (1959); 7 (3) 1: 1-160 (1961); 7 (3) 2: 161348 (1962); 7 (3) 4: 557-816 (1966). Akademische Verlagsgesselsschaft, Greest \& Porting K. G., Leipzig.

ILTIS, A., F. RISACHER \& S. SERVANT-VILDARY. 1984. Contribution á l'etude hydrobiologique des lacs salé du sud de l'altiplano bolivien. Rev. Hydrobiol. trop. 17: 259-273.

JAMES, F. \& M. C. CULLOCH. 1990. Multivariate 
analysis in Ecology and Systematics: Panacea or Pandora 'Box? Annu. Rev. Ecol. Syst. 21: 129-66.

KRAMMER, K. \& H. LANGE-BERTALOT. 1986. Bacillariophyceae 1. Teil: Naviculaceae. In: ETTL, H., J. GERLOFF, H. HEYNIG \& D. MOLLENHAUER (eds.), Süsswasserflora von Mitteleuropa, G. Fischer, Jena.

KRAMMER, K. \& H. LANGE-BERTALOT. 1988. Bacillariophyceae. 2. Teil: Bacillariaceae, Epithemiaceae, Surirellaceae. In: ETTL, H., J. GERLOFF, H. HEYNIG \& D. MOLLENHAUER (eds.), Süsswasserfloravon Mitteleuropa. G. Fischer, Jena.

KRAMMER, K. \& H. LANGE-BERTALOT. 1991. Bacillariophyceae. 3. Teil: Centrales, Fragilariaceae, Eunotiaceae. In: ETTL, H., J. GERLOFF, H. HEYNIG \& D. MOLLENHAUER (eds.), Süsswasserfloravon Mitteleuropa. G. Fischer, Jena.

KRAMMER, K. \& H. LANGE-BERTALOT. 2000. Bacillariophyceae. 3. Teil: Centrales, Fragilariaceae, Eunotiaceae. In: ETTL, H., J. GERLOFF, H. HEYNIG \& D. MOLLENHAUER (eds.), Süßwasserfloravon Mitteleuropa. G. Fischer Verlag, Jena.

KRAMMER, K. \& H. LANGE-BERTALOT. 2004. Bacillariophyceae. 4. Teil: Achnanthaceae Kritische Ergänzungenzu Navicula (Lineolatae) und Gomphonema. In: ETTL, H., J. GERLOFF, H. HEYNIG \& D. MOLLENHAUER (eds.), Süsswasserfloravon Mitteleuropa. G. Fischer, Jena.

LEGENDRE, P. \& L. LEGENDRE. 2000. Numerical Ecology. 2nd ed. Elsevier Science B.V.

LANGE-BERTALOT, H. 1997. Frankophila, Mayamaea and Fistulifera: drei neue Gattungen der Klasse Bacillariophyceae. Arch. Protistenk. 148: 65-76.

LANGE-BERTALOT, H. 1999. Neue Kombinationen von Taxa aus Achnanthes Bory (sensu lato). Iconogr. Diatomol. 6: 278-291.

LANGE-BERTALOT, H. 2001. Diatoms of Europe. 2. Navicula sensu stricto. 10 genera separated from Navicula sensu lato. Frustulia. A.R.G. GantnerVerlag, Ruggell.

LICURSI, M., N. GÓMEZ \& J. DONADELLI. 2010. Ecological optima and tolerances of coastal benthic diatoms in the freshwater-mixohaline zone of the Río de la Plata estuary. Mar. Ecol. Progr. Ser. 418: 105-117.

MAIDANA, N. I., M. S. VIGNA \& V. MASCITTI. 1998. Ficoflora de la Laguna de Pozuelos (Jujuy, Argentina) I: Bacillariophyceae. Bol. Soc. Argent. Bot. 33: 171-179.

MAIDANA, N. I., C. T. SEELIGMANN \& M. R. MORALES. 2009. Bacillariophyceae del Complejo Lagunar Vilama (Jujuy, Argentina). Bol. Soc. Argent. Bot. 44: 257-271.

MAIDANA, N. I., C. T. SEELIGMANN \& M. R.
MORALES. 2011. El género Navicula sensu stricto (Bacillariophyceae) en humedales de altura de Jujuy, Argentina. Bol. Soc. Argent. Bot. 46: 13-29.

MASCITTI, V. 1996. Partición del recurso en la comunidad de flamencos de Laguna Los Pozuelos, Jujuy, Argentina. Tesis Doctoral N ${ }^{\circ} 2904$ UBA.

MASCITTI, V. 1998. James Flamingo foraging behavior in Argentina. Colonial Waterbirds 21: 277-280.

MASCITTI V. \& M. CASTAÑERA. 1991. Avifauna y mastofauna asociada a la cuenca de la Laguna de Los Pozuelos. In: FERNÁnDEZ, J. J. \& R. TECCHI (eds.), La Reserva de la Biosfera Laguna de Los Pozuelos: un ecosistema pastoril en los Andes Centrales, pp. 51-68. PER-INBIAL, UNju Argentina.

MORRONE, J. J. 1999. Presentación preliminar de un nuevo esquema biogeográfico de América del Sur. Biogeographica 75: 1- 16.

PATRICK, R. \&. C. W. REIMER. 1966. The diatoms of the United States (exclusive of Alaska and Hawaii). Vol. 1. Acad. Nat. Sci. Philadelphia Monogr. 13: 1-688.

PATRICK, R. \&. C. W. REIMER. 1975. The diatoms of the United States (exclusive of Alaska and Hawaii). Vol. 2. Acad. Nat. Sci. of Philadelphia Monogr. 13: 1-213.

RODRIGUEZ, C. 2012. Dinámica superficial del Monumento Natural Laguna de Los Pozuelos Monitoreo de Avifauna Acuática Implicancias para su conservación. Tesis Doctoral UNSA.

ROUND, F. E., R. M. CRAWFORD \& D. G. MANN. 1990. The Diatoms. Biology and Morphology of the genera. Cambridge University Press, Cambridge.

RUMRICH, U., H. LANGE-BERTALOT \& M. RUMRICH. 2000. Diatomeen der Anden von Venezuela bis Patagonien/Tierra del Fuego. Iconogr. Diatomol. 9: 1-673.

SALUSSO, M., S. M. CAZIANI, \& S. M. MORAÑA. 1997. Distribución de plancton en relación a los caracteres limnológicos y a la avifauna de la Puna Jujeña. - CAL II.

SEELIGMANN, C. T., N. I. MAIDANA, \& M. MORALES. 2008. Diatomeas (Bacillariophyceae) de Humedales de Altura de La Provincia de JujuyArgentina. Bol. Soc. Argent. Bot. 43: 1-17.

SERVANT-VILDARY S. \& S. H. MELLO e SOUSA. 1993. Palaeohydrology of Quaternary saline Lake Ballivian (southern Bolivian Altiplano) based on diatom studies. Int. J. Salt Lake Res. 2: 69-85.

TER BRAAK, C. J. F. 1986. Canonical Correspondence Analysis: a new eigenvector thechnique for multivariated direct gradient analysis. Ecology 67: 1167-1179.

TER BRAAK, C. J. F. \& P. ŠMILAUER. 1998. CANOCO reference manual and user's guide to 


\section{A. L. González Achem et al. - Flora diatomológica en Laguna de Los Pozuelos}

CANOCO for Windows: software for canonical community ordination (Version 4). Ithaca, New York: Microcomputer Power.

VAN DAM, H., A. MERTENS \& J. SINKELDAM. 1994. A coded checklist and ecological indicator values of freshwater diatoms from the Netherlands. Netherlands J. Aquatic Ecol. 28: 117-133.
VOUILloud, A. 2003. Catálogo de diatomeas continentales y marinas de Argentina. Versión 1.0. En soporte magnético. Asociación Argentina de Ficología, La Plata.

Recibido el 28 de agosto de 2013, aceptado el 24 de febrero de 2014. 
